\title{
Autonomous wildfire hotspot detection using a fixed wing UAV
}

\section{Zhongjie Lin* and Denys Bohdanov}

Honeywell Aerospace, 3333 Unity Dr, Mississauga, ON, Canada

Email: zhongjie.lin@mail.utoronto.ca

Email: denys.bohdanov@gmail.com

*Corresponding author

\section{Hugh H.T. Liu}

Institute for Aerospace Studies,

University of Toronto,

4925 Dufferin Street, Toronto, ON, Canada

Email: liu@utias.utoronto.ca

\section{Mike Wotton}

Faculty of Forestry, University of Toronto, 33 Willcocks St., Toronto, ON, Canada

Email:mike.wotton@utoronto.ca

\begin{abstract}
This paper is concerned with wildfire hotspot detection. An autonomous unmanned aerial system (UAS) for wildfire hotspot detection missions is presented and implemented on a fixed wing low altitude short endurance (LASE) UAV. The custom built system includes the features such as hotspot detection, real-time localisation and alert of the ground control station. The on-board computer processes fire information collected by the UAV and integrates with flight data to provide a comprehensive and reliable fire detection algorithm. The proof-of-concept system has been successfully tested through flight experiments.
\end{abstract}

Keywords: hotspot detection; unmanned aerial vehicle; UAV; wildfire.

Reference to this paper should be made as follows: Lin, Z., Bohdanov, D., Liu, H.H.T. and Wotton, M. (2021) 'Autonomous Wildfire Hotspot Detection Using a Fixed Wing UAV', Int. J. Aerospace System Science and Engineering, Vol. 1, No. 1, pp.68-84.

Biographical notes: Zhongjie Lin received his $\mathrm{PhD}$ from the University of Toronto Institute for Aerospace Studies. Currently, he is a System Engineer at Honeywell Aerospace. His research interests include issues related to UAV-based wildfire monitoring and detection. 
Denys Bohdanov received his MSc in Control System Design at the University of Toronto Institute for Aerospace Studies. Currently, he is a System Engineer at Honeywell Aerospace. His research focus is vision-based target tracking and UAV controller design.

Hugh H.T. Liu is a Professor of the University of Toronto Institute for Aerospace Studies. He also serves as the Director of Natural Science and Engineering Research Council of Canada (NSERC) Collaborative Research and Training Experience (CREATE) Program on Unmanned Aerial Vehicles and Centre for Aerial Robotics Research and Education. His research interests in the area of aircraft systems and control include autonomous unmanned systems, cooperative and formation control, fault tolerant control, active control on advanced aircraft systems, as well as integrated modelling and simulations.

Mike Wotton is a Research Scientist with the Canadian Forest Service stationed at the University of Toronto. His current research focus involves developing process-based models of fuel moisture exchange, fire ignition and spread that can be applied by fire management agencies throughout Canada.

\section{Introduction}

This paper is concerned with wildfire hotspot detection. A hotspot detection mission considers detecting forest fires accurately at their early stage before they spread over large areas. An early detection of hotspots is vital for wildfire management agencies to shorten the reaction time and reduce the potential damage as well as the suppression cost. The objective for a hotspot detection mission is to detect possible fires in the target forest region fast and accurately. And the detection needs to alert the ground control personnel.

The challenges of wildfire hotspot detection are two folds. First, the target forest areas for surveillance are usually unmanaged, remote and large. Therefore, a hotspot detection system is required to have the capability of covering large areas. Second, the optical and the infrared radiation intensity of a hotspot is usually weak. The weak signal intensity poses a challenge for an accurate detection.

Conventional hotspot detection methods involve patrolling a target area by fire fighters or monitoring the area from fire watch towers. With the rapid advance of remote sensing techniques, wildfire hotspot detection can be achieved by satellite, automatic ground detection stations, aerial surveys, wireless sensor networks or UAVs according to Yuan et al. (2015). As suggested by Rauste et al. (1997), satellites have been employed for wildfire detection missions. In the work of Kelhä et al. (2003) and Gautam et al. (2008), the advanced very high resolution radiometer (AVHRR) satellite was utilised for wildfire detection. In addition, the moderate resolution imaging spectroradiometer (MODIS) satellite was proposed for fire detection in the work of Nakau et al. (2006). Satellite surveillance can provide large field of view directly from top of the fire. However, it may not give an accurate assessment due to the long sensing distance. In addition, its obvious long cycle of coverage does not provide real-time information. 
To further improve the wildfire detection and monitoring performance, ground automatic wildfire detection systems were developed to monitor the target area with high resolution and short sampling intervals. Based on infrared sensors, optical sensors or ion sensors, the ground systems can either obtain the temperature variation or detect the smoke releasing of wildfires. In the work of Arrue et al. (2000), the Bosque system, which is based on infrared cameras, was proposed for wildfire detection. The LIDAR system, which can detect plume under the situation that visual cameras cannot properly operate, was considered for wildfire detection in the work of Krider et al. (1980), Utkin et al. (2003) and Lavrov et al. (2006). Two image-based wildfire detection systems, the ForestWatch system and the FireWatch system, were compared with conventional tower observers in terms of detection rate and reporting time in the work of Matthews et al. (2012). The results from the experiment suggested that the detection of these systems was slower and less reliable than by a trained human observer. In addition, as suggested by Merino et al. (2012), the ground wildfire detection systems were limited by their small coverage range.

Wireless sensor networks (WSN) technology was also proposed by Son et al. (2006), Hartung et al. (2006), Hefeeda and Bagheri (2007) and Lloret et al. (2009) for wildfire hotspot detection missions. By deploying a mesh network of sensors to the target forest area, the WSN can detect the fire through temperature/humidity variation or visual signals. The sensors can then report the detected information to the base station through routing protocols of the internet. The WSN methods can provide accurate information with short delay. However, as suggested by Alkhatib (2014), the localisation of the sensors, the full coverage for a large forest area and the network life span are practical challenges for WSN-based hotspot detection approaches.

By navigating on top of the target forest area and reporting the potential hotspot by direct observations, piloted aerial surveillance, such as airborne wildfire intelligence system (AWIS), which was discussed by Campbell et al. (2002), can provide a good coverage and accuracy in a relatively short period cycle. It is, however, a tedious and dangerous task for pilots.

Using unmanned aerial vehicles (UAVs) that are equipped with proper sensors to fly over the forest area autonomously and detect hotspots automatically is proposed as an alternative solution. As discussed by Wilson and Davis (1988), the US Forest Services conducted the earliest application of UAV-based forest wildfire information gathering in 1961. As mentioned in the work of Yuan et al. (2015); Ambrosia and Zajkowski (2015), the 'Firebird 2001' UAV was utilised for wildfire imaging with an on-board imaging system in 1996. As presented in the work of Ambrosia (2002), the First Response Experiment (FiRE) project in the USA demonstrated the feasibility of applying a high altitude long endurance (HALE) Altus UAV for wildfire fighting missions. As discussed in the work of Merlin (2009), the NASA 'Ikhana' and 'Altair' UAVs were utilised for wildfire imaging missions in the Western US. These experiments and proposed methodologies demonstrated the potential of utilising UAVs for wildfire monitoring and detection missions. Furthermore, as proposed by Ren and Beard (2008), LASE UAVs were expected to be a key technology to further enhance the performance of wildfire detection. In the work of Merino et al. (2012, 2015), a team of LASE UAVs were utilised for wildfire detection and monitoring missions. Unmanned helicopters were utilised to verify the effectiveness of the algorithm. The method required the vehicles to hover at fixed locations in order to constantly monitoring the regional wildfire status. However, for a hotspot detection mission, instead of constant monitoring at a fixed 
location, the capability of large area surveillance, accuracy and real-time feedback are key factors that influence the mission performance.

In this paper, we present a UAV-based hotspot detection solution with novel features. Real-field flight experiments were conducted to verify the effectiveness of the designed system. First, the system is designed to detect wildfire hotspots with a fixed wing LASE UAV. A prescribed trajectory is designed for a mission to ensure the coverage of the target area. With the thermal camera and the on-board computer, the real-time hotspot information is automatically detected and processed. Second, based on the real-time information relay through the on-board radio transmitters, the online hotspot alerting and localisation are performed at the ground station to obtain an accurate and real-time hotspot situation assessment.

The rest of this paper is organised as follows: the fire detection system is discussed in Section 2. The detailed functionalities of the on-board system and the ground station are elaborated. In Section 3, field experiments are described and the results are presented. Concluding remarks are presented in Section 4.

\section{Fire detection system}

The overall architecture of the hotspot detection system is demonstrated in Figure 1. The UAV travels along the prescribed trajectory to swap the entire target forest area. The hotspot information is detected by the on-board sensor of the UAV. The real-time UAV and hotspot information are transmitted to the ground station for analysis and mapping.

Figure 1 The conceptual illustration of the wildfire hotspot detection system (see online version for colours)

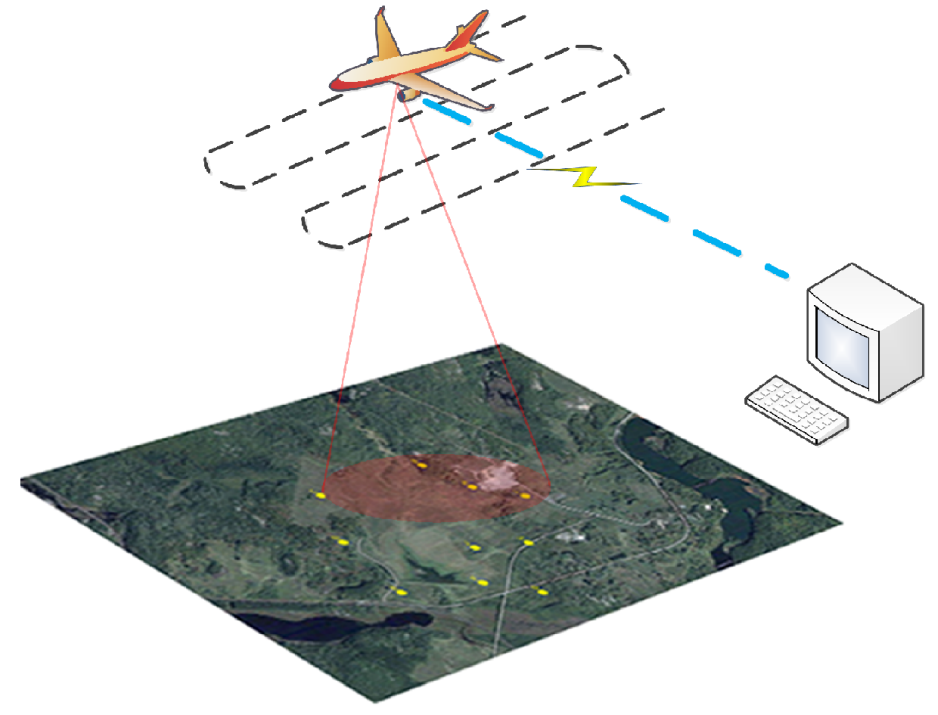




\subsection{The functionality of the on-board system and the ground station}

The wildfire hotspot detection system is composed of a UAV and a ground station. The payloads of the UAV are a thermal camera and an on-board computer. The UAV is responsible for pre-processing the information that is obtained from the thermal camera, geo-tagging the thermal image and interfacing with the ground station. The ground station is designed for post-analysis of the collected information.

Figure 2 Temperature distribution of a thermal sampling (see online version for colours)

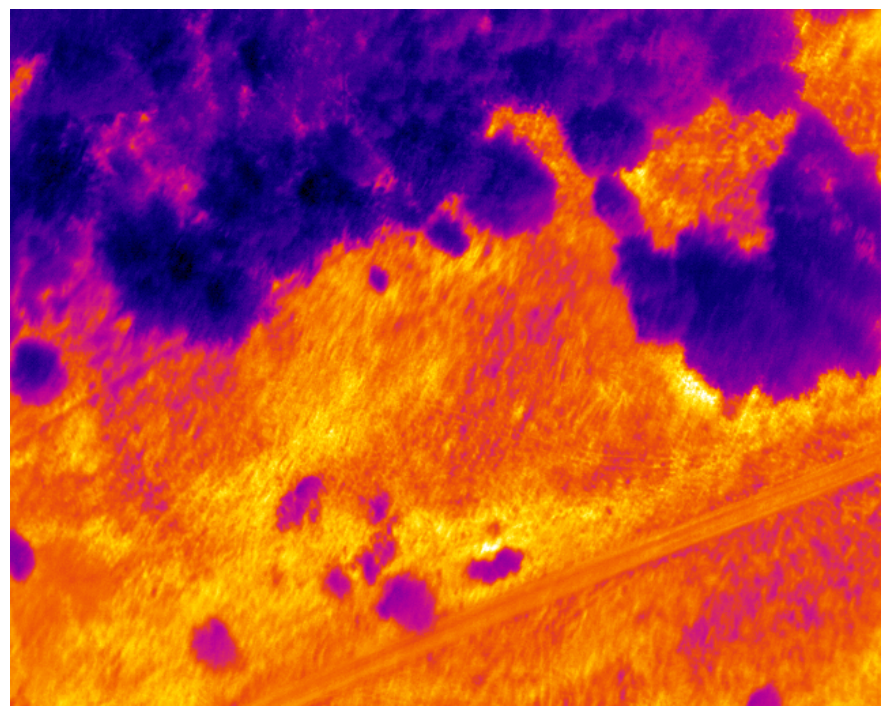

Figure 3 Thermal image after thresholding due to a valid detection of hotspot (see online version for colours)

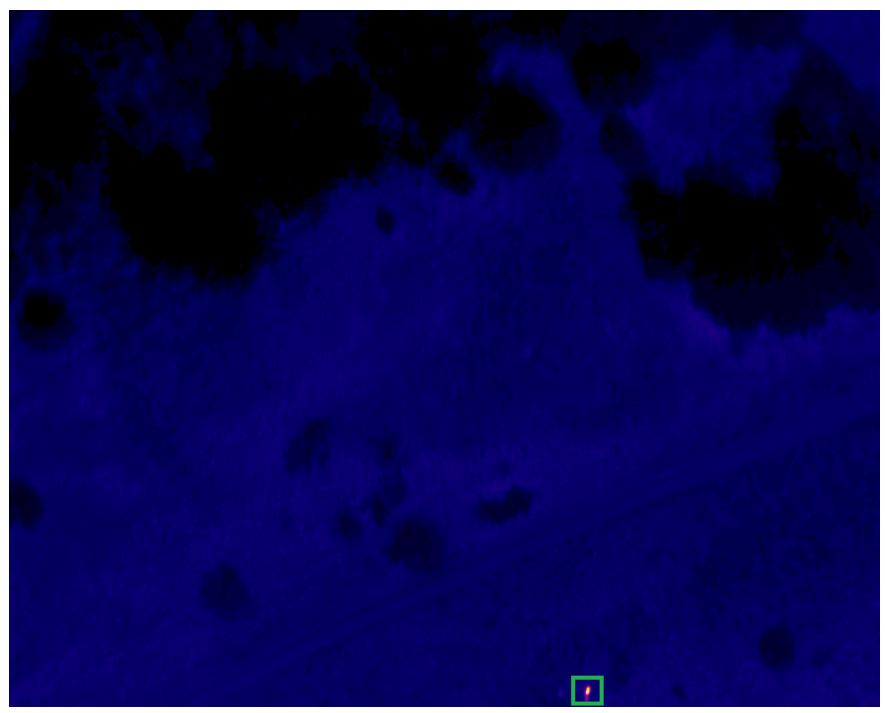


After the thermal camera takes a sampling, the on-board computer converts the raw signal values into temperature values. Figure 2 demonstrates the temperature distribution of a thermal sampling, in which different temperature values are reflected with different colors. With the converted temperature values, the system conducts a thresholding process in order to extract the desired hotspot information and generate a system alert. The thresholded output is illustrated in Figure 3.

In addition, the on-board computer synchronises the GPS and IMU information with each obtained thermal image. The synchronised data is sent to the ground station at the end of each process circle. The GPS and IMU information updates every 1 second. The thermal data is received at $4 \mathrm{~Hz}$ when no hotspots are detected and $8 \mathrm{~Hz}$ when the thermal camera captures a hotspot. The detailed on-board software architecture is demonstrated in Figure 4.

Figure 4 The architecture for the on-board system

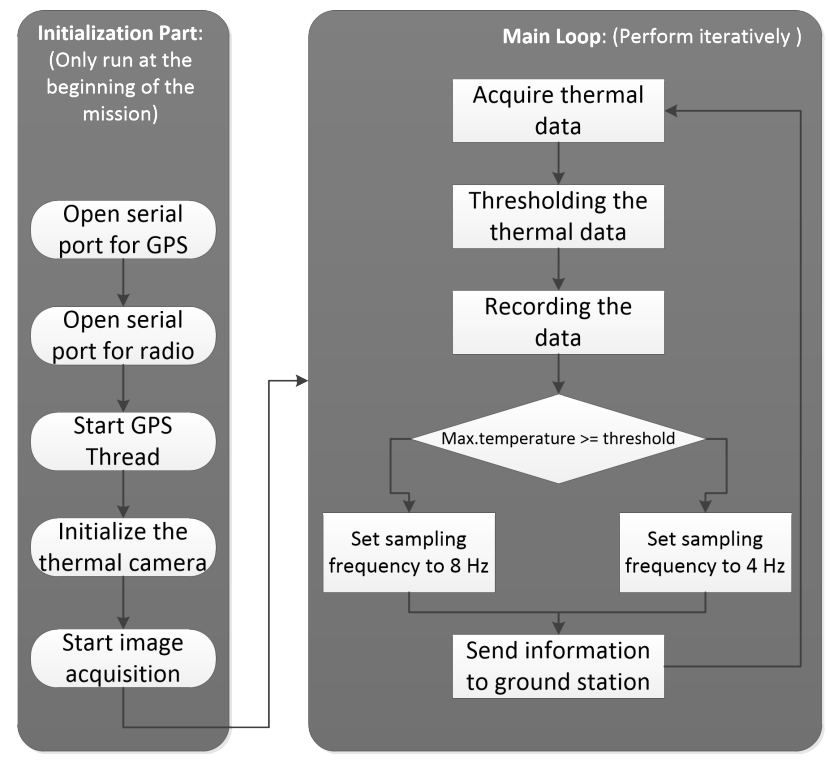

The ground station of the system is composed by two parts as the ground control station and the ground mapping station. The ground control station is responsible for generating the prescribed UAV trajectory and updating vehicle information; the ground mapping station is designed to display UAV and hotspot positions. Before the mission, the UAV trajectory is designed to cover the target forest area and uploaded to the UAV. During the mission, the ground control station is designed to update the real-time GPS and IMU information of the vehicle continuously. The ground mapping station is responsible for hotspot position rectification as well as displaying the hotspots and UAV information on the map of the field. The geo-tagged thermal information of the hotspots and the UAV positions are received through an independent radio transmitter. In Figure 6, the overall structure of the ground mapping station is demonstrated. The interfacing mechanism between the UAV and the ground station is demonstrated in Figure 5. 
Figure 5 Overall software architecture for UTIAS-Brican wildfire monitoring project (see online version for colours)

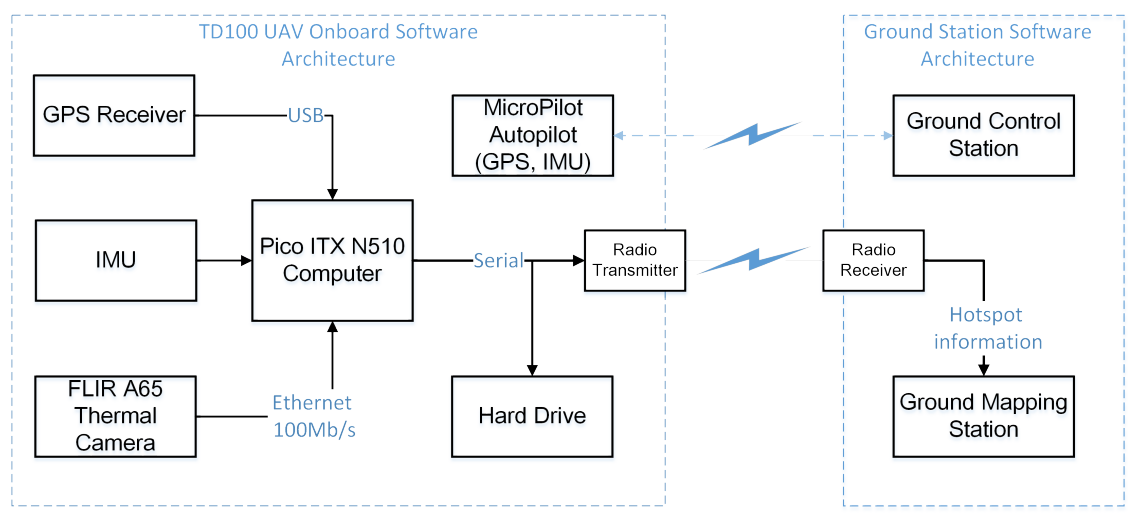

Figure 6 The code flow for the ground mapping station system

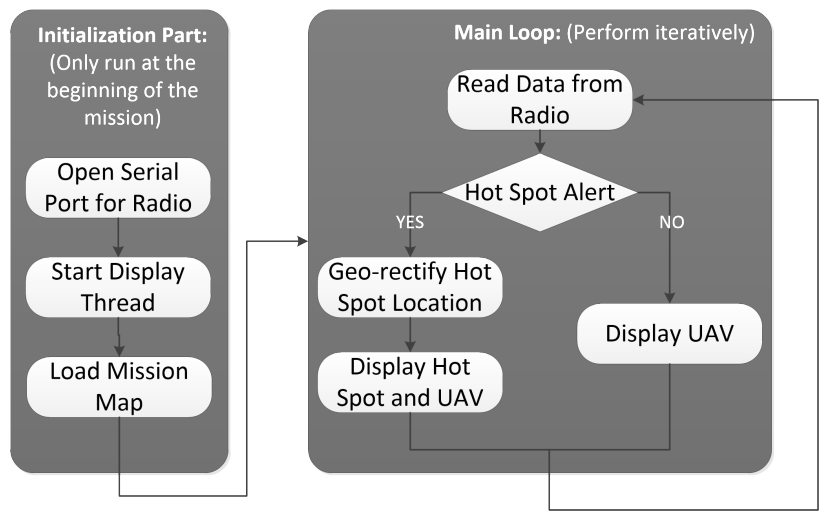

\subsection{Hotspot position rectification}

The purpose of hotspot position rectification is to utilise the online information from the UAV to estimate the accurate position of the detected hotspots. The geometry of the problem is illustrated in Figure 7.

The altitude of the UAV $h$ can be obtained by the on-board barometer sensor. The GPS location of the vehicle $H$ is obtained from the on-board GPS unit. Since the thermal camera is attached to the UAV, the body frame of the UAV also represents the camera frame.

$$
\boldsymbol{C}_{\boldsymbol{b v}}=\left(\begin{array}{ccc}
c_{\theta} c_{\psi} & c_{\theta} s_{\psi} & -s_{\theta} \\
s_{\phi} s_{\theta} c_{\psi}-c_{\phi} s_{\psi} & s_{\phi} s_{\theta} s_{\psi}+c_{\phi} c_{\psi} & s_{\phi} c_{\theta} \\
c_{\phi} s_{\theta} c_{\psi}+s_{\phi} s_{\psi} & c_{\phi} s_{\theta} s_{\psi}-s_{\phi} c_{\psi} & c_{\phi} c_{\theta}
\end{array}\right)
$$


with $c_{(\cdot)}=\cos (\cdot)$ and $s_{(\cdot)}=\sin (\cdot)$. The $C_{\boldsymbol{b} v}$ represents the transformation matrix from the vehicle frame to the body frame. If we denote the $\overrightarrow{G J}$ vector as $\overrightarrow{J^{b}}$ in the body frame and $\overrightarrow{J^{v}}$ in the vehicle frame, we have

$$
\left.\begin{array}{l}
\overrightarrow{J^{v}}=\left[0,0, h^{\prime}\right] \\
\overrightarrow{J^{b}}=C_{\boldsymbol{b} v} \overrightarrow{J^{v}}
\end{array}\right\} \rightarrow h^{\prime}=\frac{f}{c_{\phi} c_{\theta}}
$$

Figure 7 The illustration for the hotspot position rectification algorithm (see online version for colours)

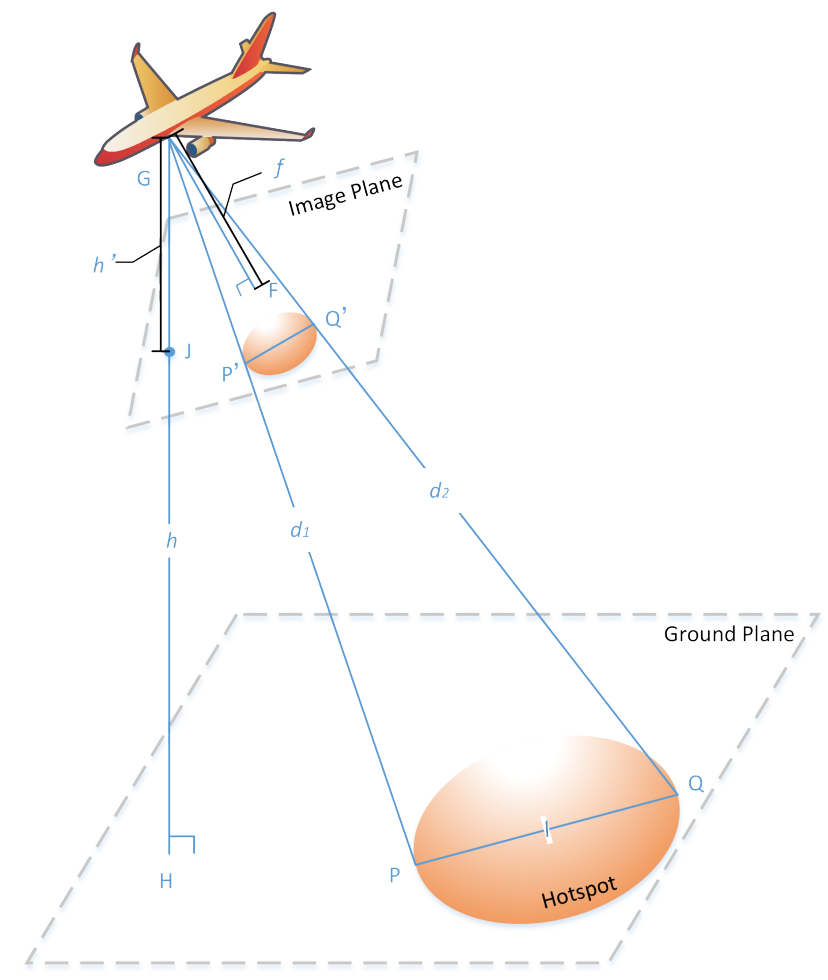

Further, if denote $\overrightarrow{P^{\prime b}}$ as $\overrightarrow{G P^{\prime}}$ in the body frame and $\overrightarrow{P^{v}}$ as the $\overrightarrow{G P}$ in vehicle frame, the $\overrightarrow{P^{v}}$ can be represented by

$$
\overrightarrow{P^{v}}=C_{b v}^{-1} \frac{\overrightarrow{P^{\prime b}}}{\left|\overrightarrow{P^{\prime b}}\right|} \frac{h}{\cos \alpha}
$$

with

$$
\alpha=\angle P^{\prime} G J=\arccos \left(\frac{\overrightarrow{P^{\prime b}} \overrightarrow{J^{b}}}{\left|\overrightarrow{P^{\prime b}}\right|\left|\overrightarrow{J^{b}}\right|}\right)
$$

The vector $\overrightarrow{H P}$ is $\overrightarrow{H P}=\overrightarrow{G P}-\overrightarrow{G H}$. Therefore, the position of the hotspot $P$ can be obtained. 


\section{Field experiments}

\subsection{Position rectification verification}

In order to verify the performance of the position rectification algorithm, an octo-copter with a RGB camera is utilised. The experiment requires the octo-copter taking pictures at different altitude. The RGB camera involved in the experiment is Sony ILCE-6000 camera. The experiment parameters and results are demonstrated in Tables 1 and 2. The ground target of the experiment is presented in Figure 8.

Table 1 Altitude estimation experiment parameters

\begin{tabular}{lc}
\hline Pixel size & $3.92 \mathrm{E}-6 \mathrm{~m}$ \\
Focus length & $2 \mathrm{E}-2 \mathrm{~m}$ \\
Dimensions & $6,000 \times 4,000$ \\
Resolution & $350 \mathrm{dpi}$ \\
Target GPS & $(43.782115,-79.465815)$ \\
\hline
\end{tabular}

Table 2 Position estimation results

\begin{tabular}{lccc}
\hline Vehicle position & Alt. (Baro) & V-T Dist. & Error \\
\hline $43.782156,-79.465789$ & $11.74 \mathrm{~m}$ & $6.496 \mathrm{~m}$ & $1.42 \mathrm{~m}$ \\
$43.782152,-79.465787$ & $21.59 \mathrm{~m}$ & $6.841 \mathrm{~m}$ & $0.98 \mathrm{~m}$ \\
$43.782153,-79.465782$ & $49.50 \mathrm{~m}$ & $7.145 \mathrm{~m}$ & $0.03 \mathrm{~m}$ \\
$43.782156,-79.465787$ & $77.09 \mathrm{~m}$ & $6.654 \mathrm{~m}$ & $0.62 \mathrm{~m}$ \\
$43.782153,-79.465789$ & $101.51 \mathrm{~m}$ & $6.633 \mathrm{~m}$ & $0.61 \mathrm{~m}$ \\
\hline
\end{tabular}

Figure 8 The ground target of the experiment (see online version for colours)

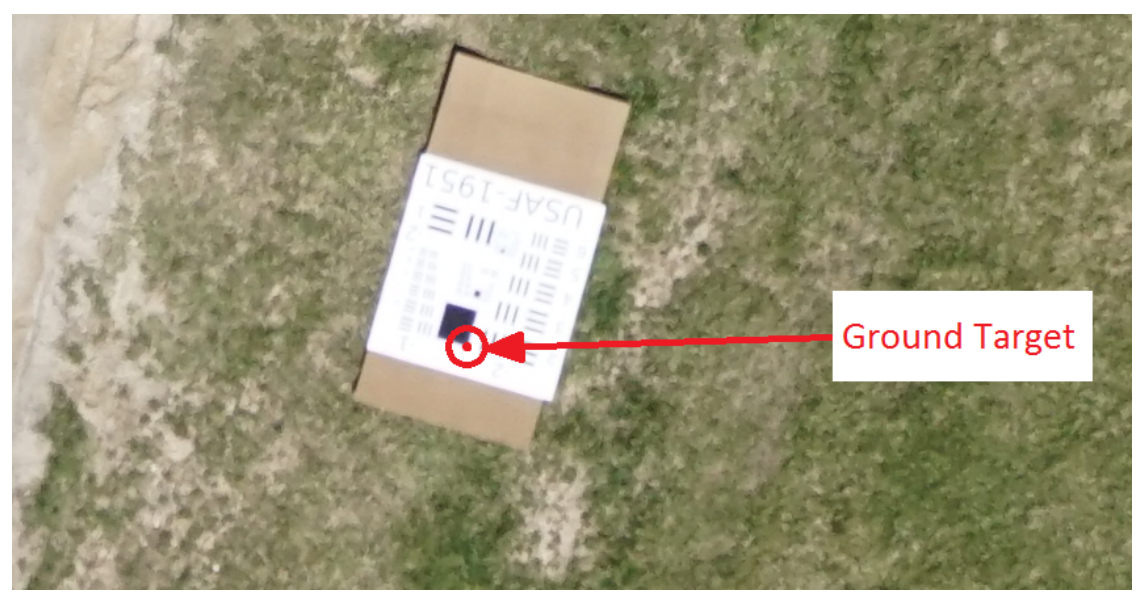

In the experiment, five measurements are taken at different altitude as shown in Table 2. The first column of the table shows the GPS position of the octo-copter and the second column of the table is the altitude of the vehicle from barometer readings. The data of the third column is the distance between the vehicle and the target. The fourth 
column demonstrates the distance error between the corrected hotspot position and the actual position of the ground target. The results suggest that the accuracy for the position estimation is improved with the rectification algorithm.

\subsection{Burwash flight test}

This experiment is a collaboration between the Flight Systems and Control (FSC) group at University of Toronto Institute for Aerospace Studies and Brican Flight systems Inc., which is a Canadian company specialised in UAV manufacturing and services. FSC was responsible for algorithm design and payload implementing. The payload of the UAV is composed of a thermal camera for information gathering and an on-board computer for image processing and data recording. In addition, the ground station is also designed by FSC for mapping and post-analysis of the detected hotspots in real-time. Brican provided the unmanned aerial vehicle platform. The experiment also received great help from the Ministry's Aviation, Forest Fire and Emergency Services (AFFES).

\subsubsection{The UAV and payload}

The fixed wing Brican TD100 UAV is designed and customised for the hotspot detection mission by Brican. Based on the catapult-launch system, the vehicle can be deployed to the target area efficiently. The wing span of the vehicle is $488.95 \mathrm{~cm}$ and the vehicle length is $200.25 \mathrm{~cm}$. The payload capability of the vehicle is around $9.1 \mathrm{~kg}$ and its cruise speed is $83 \mathrm{kph}$. The vehicle can continuously conduct the mission for approximately 4 hours. The designed vehicle is also capable of tracking a prescribed trajectory autonomously with the on-board autopilot system and providing real-time GPS and IMU information.

The payload of the UAV for the hotspot detection mission is composed of a FLIR A65 thermal camera unit for acquisition of radiometric data and a Pico ITX LP-170H on-board computer for image processing and communication. The FLIR A65 thermal camera can provide a wide range of temperature measurement from $-40^{\circ} \mathrm{C}$ to $+550^{\circ} \mathrm{C}$ with a derivation around $\pm 5^{\circ} \mathrm{C}$ or $\pm 5 \%$ of the reading. The resolution of the camera is $640 \times 512$. The maximum sampling frequency of the camera is $8 \mathrm{~Hz}$ with the detector time constant as $12 \mathrm{~ms}$. Since the mission altitude of the UAV is around $450 \mathrm{~m}$, the approximate coverage for a 4 hour mission is $53 \mathrm{~km}^{2}$ and a pixel distance on the image plane represents $0.25 \mathrm{~m}$ on ground.

\subsubsection{Mission details}

Test flights were performed near Sudbury at the Burwash Training Site for fire fighters training and prescribed fire fighting exercises. The experiment received a lot of support from AFFES which coordinates forest fire detection, monitoring, suppression, public information and education services for Ontario. AFFES played a huge role during the test flights by providing field testing site, staff support, as well as evaluation of test results. The UTIAS-Brican hotspot detection flight testing at the Burwash Training Site was scheduled on 23-24 May of 2013. The designated flight testing area and the hotspot positions are demonstrated in Figure 9. The hotspot locations were randomly selected and distributed separately in the field as listed in Table 3. Ten hotspots were placed 
throughout the designated area by the MNR fire crews for fire detection and monitoring purpose. A special flight operation certificate was obtained prior to the flight test.

Figure 9 The hotspot location and distribution in the designated flight testing area (see online version for colours)

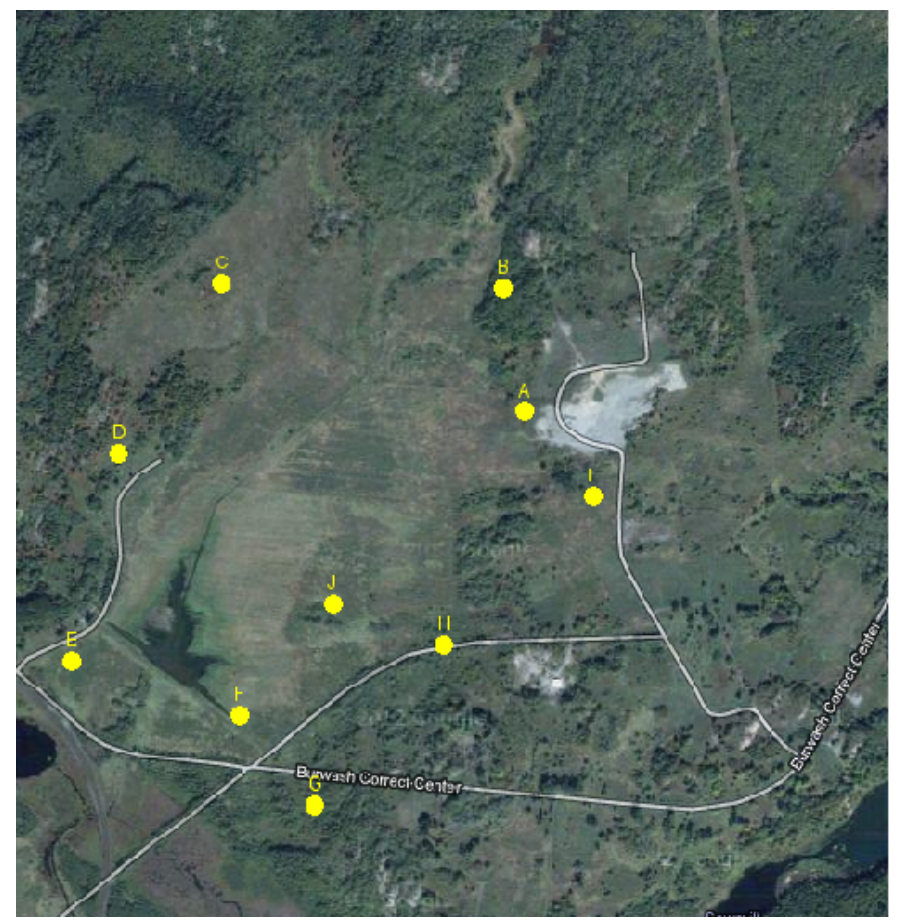

Table 3 Hotspot locations and types

\begin{tabular}{lcccc}
\hline Spots & GPS $(\mathrm{DD})$ & Uncor. Err. $(\mathrm{m})$ & Cor. Err. $(\mathrm{m})$ & Type \\
\hline Alpha & $46.2468-80.8525$ & 62 & 3.13 & Bushline \\
Bravo & $46.2491-80.8531$ & 20 & 3.95 & Cover \\
Charlie & $46.2492-80.8609$ & 79 & 3.97 & Bushline \\
Delta & $46.2460-80.8637$ & 52 & 0.34 & Cover \\
Echo & $46.2421-80.8650$ & 74 & 3.98 & Field \\
Foxtrot & $46.2411-80.8604$ & 40 & 1.78 & Field \\
Golf & $46.2394-80.8583$ & $\mathrm{NA}$ & $\mathrm{NA}$ & Cover \\
Hotel & $46.2424-80.8547$ & 20 & 5.93 & Cover \\
India & $46.2452-80.8506$ & 35 & 1.78 & Cover \\
Juliette & $46.2432-80.8578$ & 27 & 2.50 & Field \\
\hline
\end{tabular}

The hotspots are $60 \mathrm{~cm} \times 30 \mathrm{~cm}$ pans with charcoal as fuel and the average temperature of the hotspots during the flight is around $450^{\circ} \mathrm{C}$. The position of the hotspot Golf was changed in the mission. Three different set-ups of the hotspots are considered during the mission in order to simulate different situation of a real wildfire hotspot detection mission: 
- bushline hotspots: the hotspot pans are setting besides bushes to simulate the situation that a fire occurs in a bush area

- $\quad$ open hotspots: the hotspot pans are setting on open areas to simulate a grassland wildfire

- covered hotspots: the pans of the hotspots are covered by vegetation and canopy.

The flight path of the aircraft follows the standard Zamboni pattern which ensures that the entire area of interest is covered as demonstrated in Figure 10. The flight path was selected by assuming no prior knowledge of the hotspot locations. The total area coverage and the field of view of the thermal camera were considered to design the flight path such that the UAV can sweep the entire area of interest and there are $50 \%$ overlap in the thermal images. Figures 11, 12, 13 and 14 demonstrate the thermal images of the detected hotspots (Alpha, Charlie, Echo and India).

Figure 10 The flight path for the hotspot detection mission (see online version for colours)

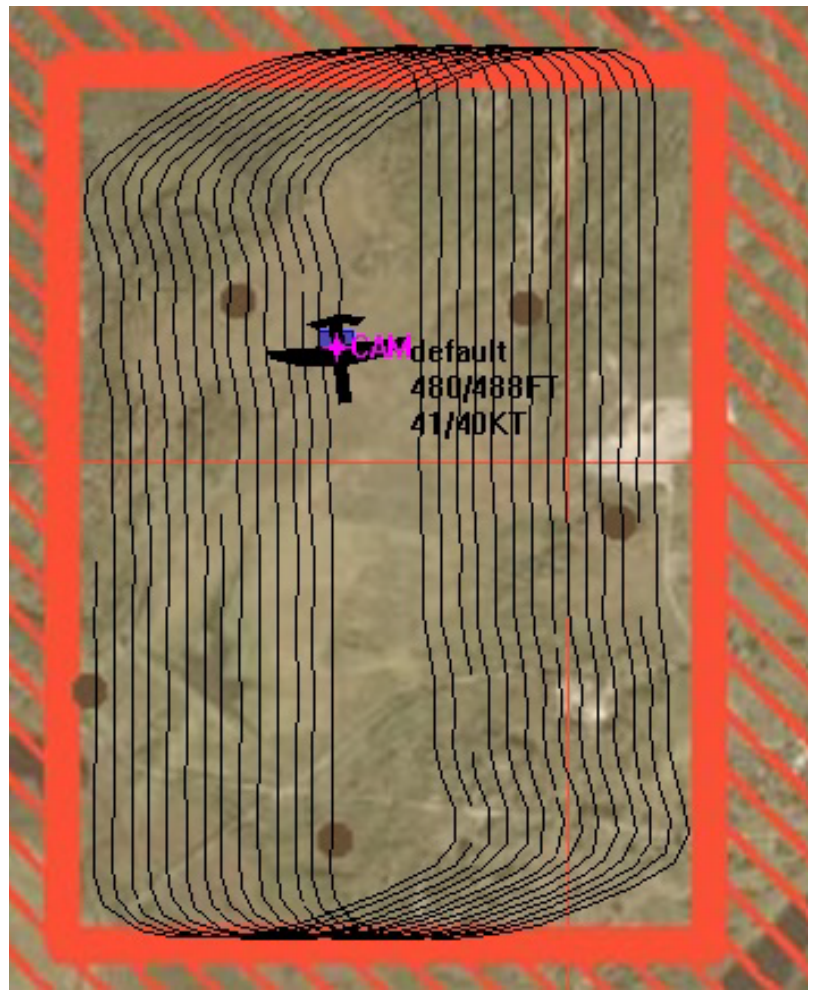

Figure 15 shows all the hotspots detected by the UAV during the 1 hour and 15 min operation with not position correction such that the position of each hotspot is assumed to be the same as the position of the UAV as it was flying over the hotspot. From the result, 9 out of 10 hotspots were detected and reported to the ground station in real-time. The hotspot Golf was not detected because the UAV did not cover that area during the operation. 
Figure 11 Hot-spot Alpha (bushline) (see online version for colours)

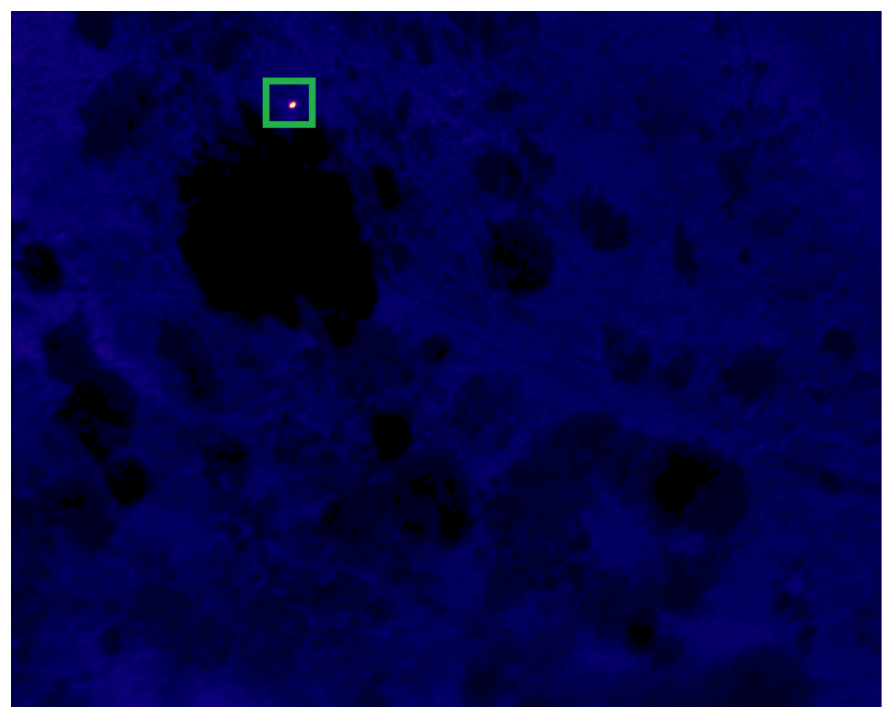

Figure 12 Hot-spot Charlie (bushline) (see online version for colours)

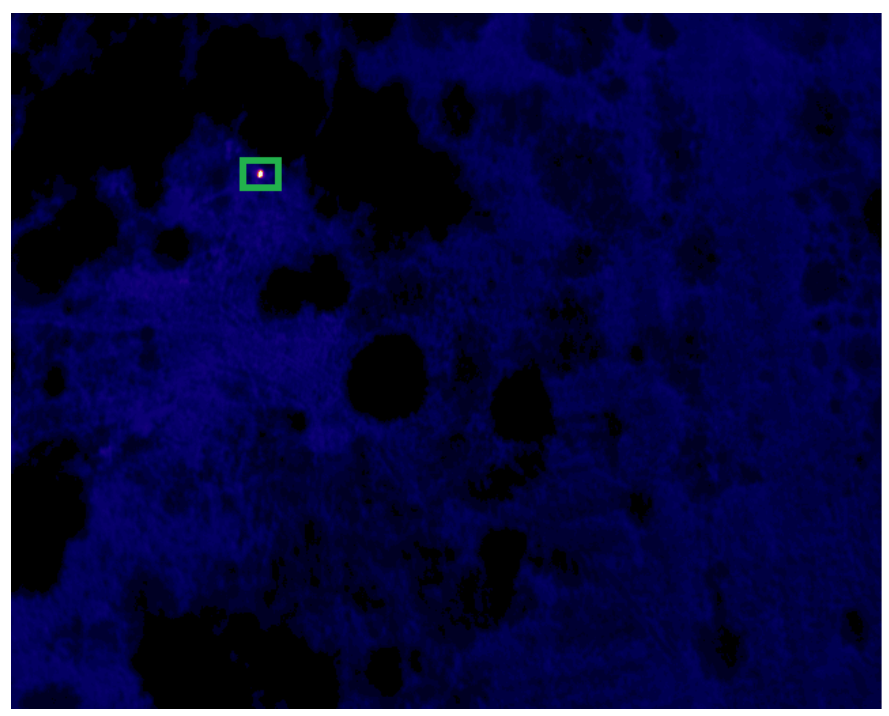

Three false positives were identified during the mission. The post-flight analysis suggested that the false positives were caused by a crew member riding through the area on an ATV. The reported temperature of the false alarms was $85^{\circ} \mathrm{C}$ on average coming from the engine of the ATV. All three false positives were generated by the same ATV recorded at different places on different passes. Figure 16 shows the rectified positions of each hotspot and Table 3 summarises the detection results. The GPS position of each hotspot is demonstrated in the second column of the table. Since the UAV provided multiple detections for each hotspot at different time instants and passes, the column of the uncorrected error represents the error by taking the averaged vehicle position as the 
hotspot position without the position rectification. The error of corrected hotspot position is presented in the fourth column of Table 3. The averaged error of the uncorrected errors is 45 metres and after the rectification process, the position error reduces to 3 metres. The results demonstrate the potential of this method for finding accurate hotspot locations.

Figure 13 Hot-spot Echo (field) (see online version for colours)

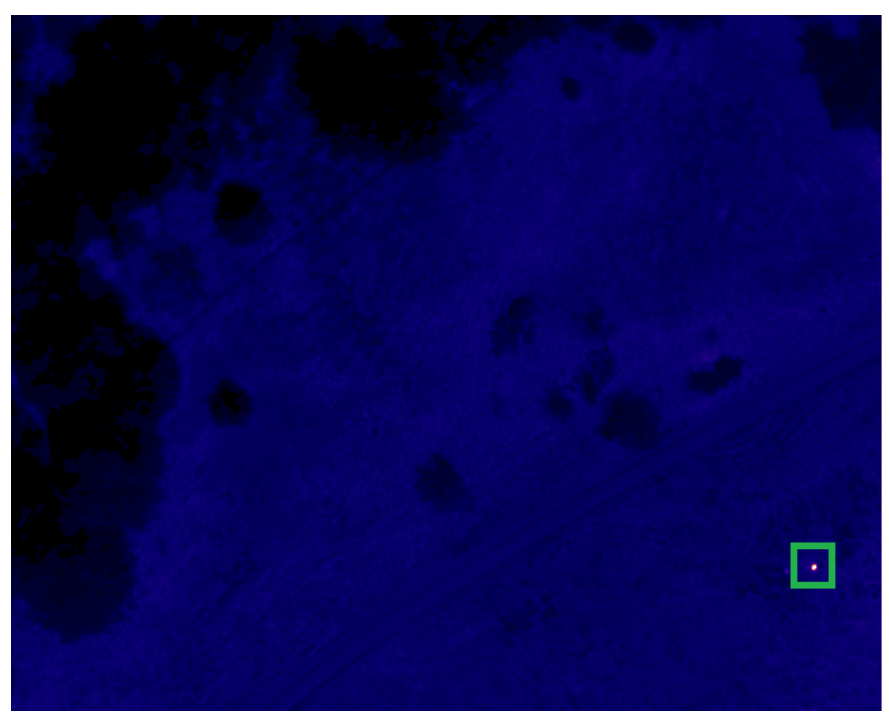

Figure 14 Hot-spot India (field) (see online version for colours)

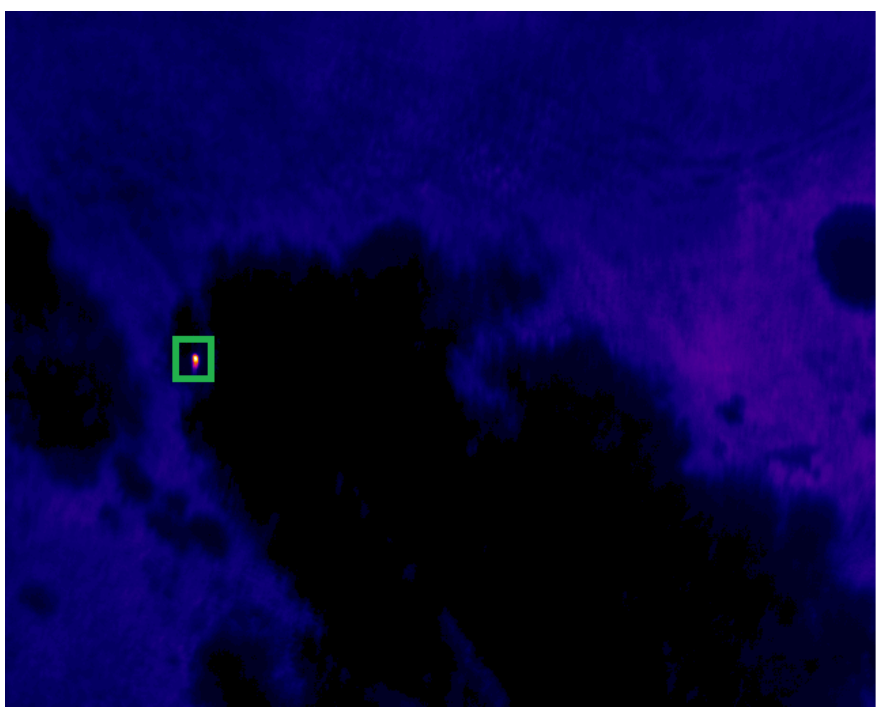


Figure 15 The reported vehicle position when a hotspot is detected (see online version for colours)

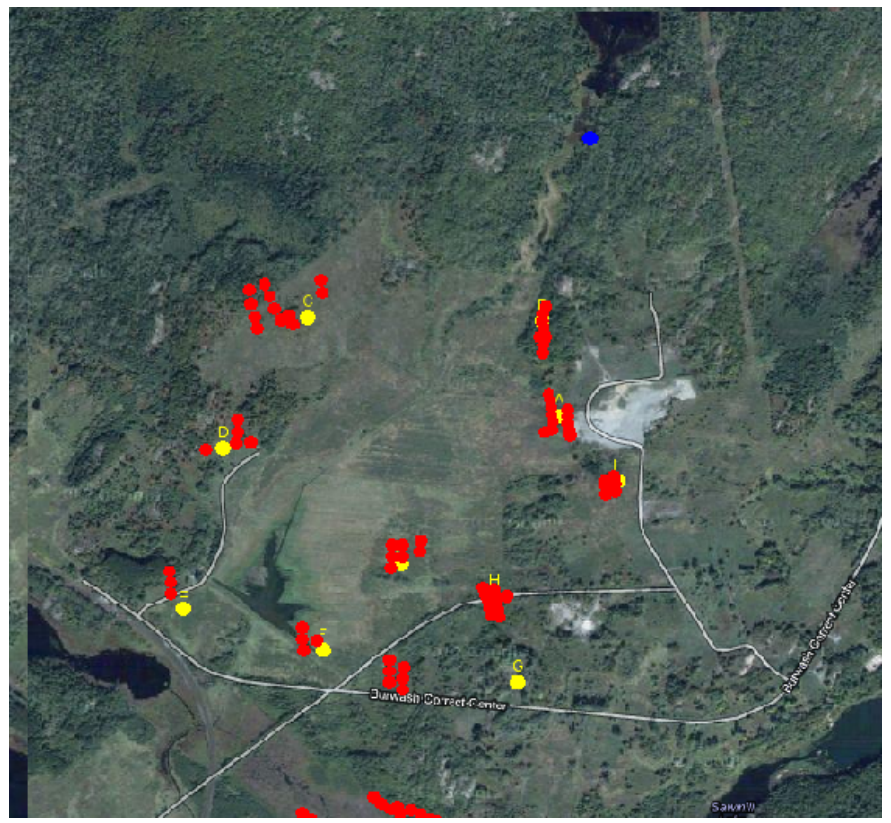

Figure 16 The hotspot detection result of the flight test (see online version for colours)

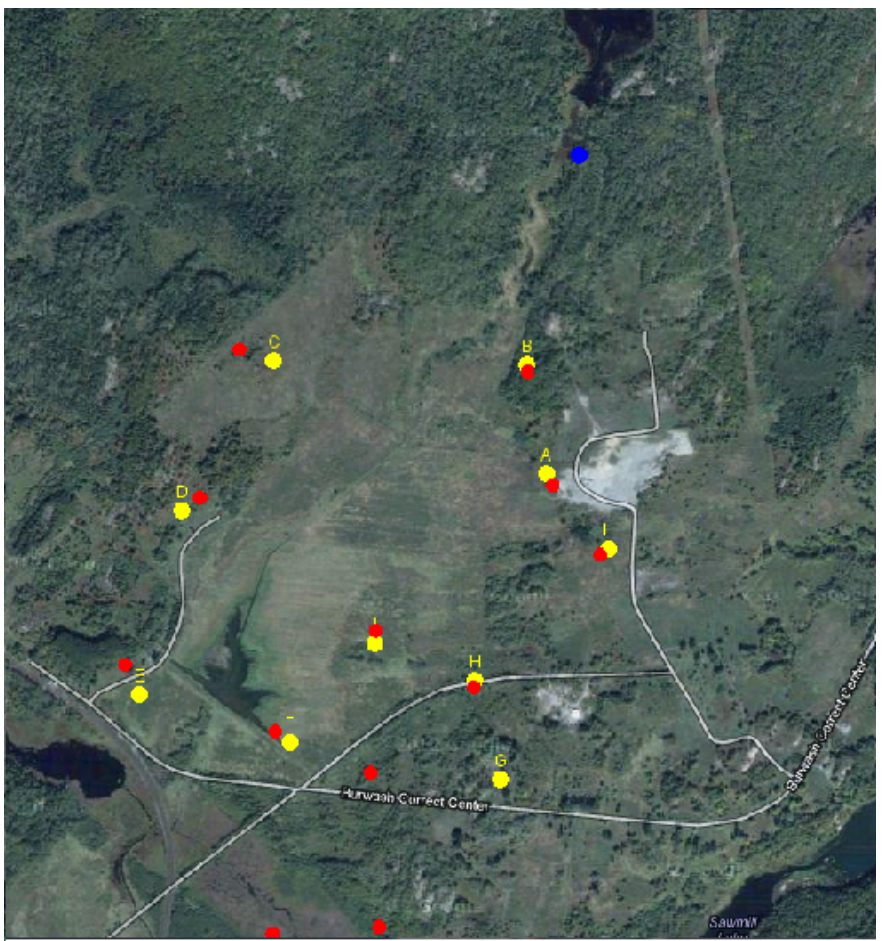




\section{Conclusions and future works}

A wildfire hotspot detection system and its experimental verification are presented in this paper. The system platform is introduced and the detailed architecture concerning the on-board system and the ground station are presented. Real-field experiments are conducted to verify the effectiveness of the designed system and the experiment results demonstrate the potential of the system for detecting hotspots.

In the future works, we consider to utilise multiple UAVs to monitor the target forest area in a cooperative manner to improve the mission coverage and efficiency. In addition, the research concerning online trajectory planning will be conducted to let UAVs decide their individual trajectories according to the real-time fire situation.

\section{References}

Alkhatib, A.A. (2014) 'A review on forest fire detection techniques', International Journal of Distributed Sensor Networks, Vol. 10, No. 3, p.597368.

Ambrosia, V. (2002) 'Remotely piloted vehicles as fire imaging platforms: the future is here', Wildfire Magazine, Vol. 11, No. 3, pp.9-16.

Ambrosia, V.G. and Zajkowski, T. (2015) 'Selection of appropriate class UAS/sensors to support fire monitoring: experiences in the United States', Handbook of Unmanned Aerial Vehicles, pp.2723-2754, Springer, Dordrecht, the Netherlands.

Arrue, B.C., Ollero, A. and de Dios, J.M. (2000) 'An intelligent system for false alarm reduction in infrared forest-fire detection', IEEE Intelligent Systems and Their Applications, Vol. 15, No. 3, pp.64-73.

Campbell, D., Born, W.G., Beck, J., Bereska, B., Frederick, K. and Hua, S. (2002) 'Airborne wildfire intelligence system: a decision support tool for wildland fire managers in Alberta', Thermosense XXIV, Vol. 4710, pp.159-171, International Society for Optics and Photonics.

Gautam, R.S., Singh, D. and Mittal, A. (2008) 'An efficient contextual algorithm to detect subsurface fires with NOAA/AVHRR data', IEEE Transactions on Geoscience and Remote Sensing, Vol. 46, No. 7, pp.2005-2015.

Hartung, C., Han, R., Seielstad, C. and Holbrook, S. (2006) 'FireWxNet: a multi-tiered portable wireless system for monitoring weather conditions in wildland fire environments', Proceedings of the 4th International Conference on Mobile Systems, Applications and Services, pp.28-41, ACM.

Hefeeda, M. and Bagheri, M. (2007) 'Wireless sensor networks for early detection of forest fires', IEEE International Conference on Mobile Adhoc and Sensor Systems, 2007. MASS 2007, pp.1-6, IEEE.

Kelhä, V., Rauste, Y., Häme, T., Sephton, T., Buongiorno, A., Frauenberger, O., Soini, K., Venäläinen, A., Miguel-Ayanz, J.S. and Vainio, T. (2003) 'Combining AVHRR and ATSR satellite sensor data for operational boreal forest fire detection', International Journal of Remote Sensing, Vol. 24, No. 8, pp.1691-1708.

Krider, E., Noggle, R., Pifer, A. and Vance, D. (1980) 'Lightning direction-finding systems for forest fire detection', Bulletin of the American Meteorological Society, Vol. 61, No. 9, pp.980-986.

Lavrov, A., Utkin, A.B., Vilar, R. and Fernandes, A. (2006) 'Evaluation of smoke dispersion from forest fire plumes using LIDAR experiments and modelling', International Journal of Thermal Sciences, Vol. 45, No. 9, pp.848-859.

Lloret, J., Garcia, M., Bri, D. and Sendra, S. (2009) 'A wireless sensor network deployment for rural and forest fire detection and verification', Sensors, Vol. 9, No. 11, pp.8722-8747. 
Matthews, S., Sullivan, A., Gould, J., Hurley, R., Ellis, P. and Larmour, J. (2012) 'Field evaluation of two image-based wildland fire detection systems', Fire Safety Journal, Vol. 47, No. 1, pp.54-61.

Merino, L., Caballero, F., Martínez-de-Dios, J.R., Maza, I. and Ollero, A. (2012) 'An unmanned aircraft system for automatic forest fire monitoring and measurement', Journal of Intelligent \& Robotic Systems, Vol. 65, Nos. 1-4, pp.533-548.

Merino, L., Martínez-de Dios, J.R. and Ollero, A. (2015) 'Cooperative unmanned aerial systems for fire detection, monitoring, and extinguishing', Handbook of Unmanned Aerial Vehicles, pp.2693-2722, Springer, Dordrecht, the Netherlands.

Merlin, P.W. (2009) Ikhana: Unmanned Aircraft System Western States Fire Missions, National Aeronautics and Space Administration, NASA History Office, Washington, DC, USA.

Nakau, K., Fukuda, M., Kushida, K., Hayasaka, H., Kimura, K. and Tani, H. (2006) 'Forest fire detection based on modis satellite imagery, and comparison of NOAA satellite imagery with fire fighters' information', IARC/JAXA Terrestrial Team Workshop, pp.18-23.

Rauste, Y., Herland, E., Frelander, H., Soini, K., Kuoremaki, T. and Ruokari, A. (1997) 'Satellite-based forest fire detection for fire control in boreal forests', International Journal of Remote Sensing, Vol. 18, No. 12, pp.2641-2656.

Ren, W. and Beard, R.W. (2008) Distributed Consensus in Multi-vehicle Cooperative Control, Springer, London, England.

Son, B., Her, Y-S. and Kim, J-G. (2006) 'A design and implementation of forest-fires surveillance system based on wireless sensor networks for South Korea mountains', International Journal of Computer Science and Network Security (IJCSNS), Vol. 6, No. 9, pp.124-130.

Utkin, A.B., Fernandes, A., Simões, F., Lavrov, A. and Vilar, R. (2003) 'Feasibility of forest-fire smoke detection using LIDAR', International Journal of Wildland Fire, Vol. 12, No. 2, pp.159-166.

Wilson, C.C. and Davis, J.B. (1988) Forest Fire Laboratory at Riverside and Fire Research in California: Past, Present, and Future, Gen. Tech. Rep. PSW-105, Pacific Southwest Research Station, Forest Service, US Department of Agriculture, Berkeley, Calif., 22pp.

Yuan, C., Zhang, Y. and Liu, Z. (2015) 'A survey on technologies for automatic forest fire monitoring, detection, and fighting using unmanned aerial vehicles and remote sensing techniques', Canadian Journal of Forest Research, Vol. 45, No. 7, pp.783-792. 